\title{
Robust Multi-Beam Multiplexing Design Based on a Hybrid Beamforming Structure with Nearly Equal Magnitude Analogue Coefficients
}

\author{
Junwei Zhang, Student Member, IEEE, Wei Liu, Senior Member, IEEE, Chao Gu, \\ Steven Shichang Gao, Fellow, IEEE, and Qi Luo, Senior Member, IEEE
}

\begin{abstract}
One novel design considering two practical application constraints based on the interleaved subarray architecture is proposed to achieve multi-beam multiplexing for arbitrary directions to serve corresponding users. One is an equal magnitude constraint imposed on the analogue coefficients so that beamforming can be achieved by pure phase shifters after the normalisation of magnitudes; the other one is a robust constraint against steering vector errors. Designed examples are provided to verify the effectiveness of the proposed method.
\end{abstract}

Index Terms-Interleaved subarray architecture, beam multiplexing, equal magnitude constraint, steering vector errors

\section{INTRODUCTION}

T HE hybrid beamforming structure has been a widely recognised solution for the implementation of massive MIMO and mmWave communications in 5G [1]-[11], where the analogue beamforming technique [12] and the digital beamforming technique are combined together.

One representative hybrid beamforming structure is the subaperture based hybrid beamformer [3], [5], [13]-[15], and there are mainly two types of them: the localised subarray architecture and the interleaved subarray architecture [16] [20]. Recently, a hybrid beamforming method which involves multiplexing multiple user beams was proposed [21]-[23]. One particular feature of the method is that, the number of analogue coefficients is the same as the number of antennas, independent of the number of beams generated, while the number of subarrays is the same as the number of beams.

In this work, we extend the work in [23] by considering two practical constraints. One is that the analogue coefficient of each antenna may only change its phase after the normalisation of magnitudes so that a uniform fixed-magnitude coefficient is applied to each of the received analogue signals. This will reduce the implementation complexity of the analogue beamformer significantly [24]-[30], because only phase shifts are needed to steer the multiple beams to different directions. The second constraint is related to the robustness of the system against different perturbations, such as antenna location and

J. Zhang and W. Liu are with the Department of Electronic and Electrical Engineering, University of Sheffield, Sheffield, S1 3JD, UK(e-mail: jzhang96@sheffield.ac.uk, w.liu@sheffield.ac.uk)

C. Gu is with the ECIT Institute, Queen's University Belfast, Belfast, BT3 9DT, UK(e-mail: chao.gu@qub.ac.uk)

S. Gao is with the School of Engineering and Digital Arts, University of Kent, Canterbury, CT2 7NT, UK(e-mail: s.gao@kent.ac.uk)

Q. Luo is with the School of Physics, Engineering and Computer Science, University of Hertfordshire, Hertfordshire, UK(e-mail: q.luo2@herts.ac.uk) response errors and the mutual coupling effect [31]-[34]. To improve the robustness of the designed beamformer, a normbounded error is introduced to the steering vector of the array and a corresponding constraint is placed on the overall design process. With the constraint, the difference between the desired and real beam responses generated by this robust beamformer can be controlled below an acceptable level.

Different from the traditional design for a single beam pattern with multiple main beam directions, the real challenge here is to design a common set of analogue coefficients generating independent multiple beams with each beam used by one user only, while simultaneously meeting the two required constraints. Again unlike the traditional case, which is often convex, the new problem is non-convex and a novel solution has been proposed to solve it effectively following an iterative optimisation approach.

For the remaining part, a review of the interleaved subarray architecture is presented in Sec. II. The proposed design is given in Sec. III. Design examples are provided in Sec. IV and conclusions are drawn in Sec. V.

\section{THE INTERLEAVED SUBARRAY ARCHITECTURE}

The considered interleaved subarray structure based on an N-element uniform linear array (ULA) is shown in Fig. 1, where the adjacent antenna spacing is $d$. Suppose the $\mathrm{N}$ elements of the ULA are split into $M$ interleaved subarrays. Then, each subarray consists of $\mathrm{N}_{\mathrm{s}}=\mathrm{N} / \mathrm{M}$ antennas with an adjacent antenna spacing $d_{\mathrm{m}}=\mathrm{M} d$. The phase shift between adjacent subarrays is $e^{j 2 \pi \frac{d}{\lambda} \sin \theta}$, where the direction of angle $\theta$ is measured from the broadside of the array.

The steering vector of the m-th subarray in the interleaved subarray architecture is given by

$$
\begin{aligned}
\mathbf{S}_{\mathrm{m}}(\theta)= & {\left[e^{j 2 \pi \mathrm{m} \frac{d}{\lambda} \sin \theta}, e^{j 2 \pi \frac{(\mathrm{m}+\mathrm{M}) d}{\lambda} \sin \theta}\right.} \\
& \left.\ldots, e^{j 2 \pi\left(\mathrm{m}+\mathrm{M}\left(\mathrm{N}_{\mathrm{s}}-1\right)\right) \frac{d}{\lambda} \sin \theta}\right]^{\mathrm{T}}
\end{aligned}
$$

where $[.]^{\mathrm{T}}$ is the transpose operation with $\mathrm{m} \in\{0,1, \cdots, \mathrm{M}-$ $1\}$. The beam response generated by the $m$-th subarray is

$$
P_{\mathrm{m}}(\theta)=\mathbf{w}_{\mathrm{m}}^{\mathrm{H}} \mathbf{s}_{\mathrm{m}}(\theta),
$$

where $[.]^{\mathrm{H}}$ is the Hermitian transpose and $\mathbf{w}_{\mathrm{m}}$ denotes the analogue coefficients for the m-th subarray, given by

$$
\mathbf{w}_{\mathrm{m}}=\left[w_{\mathrm{m}, 0}, w_{\mathrm{m}, 1}, \cdots, w_{\mathrm{m}, \mathrm{N}_{\mathrm{s}}-1}\right]^{\mathrm{T}} .
$$




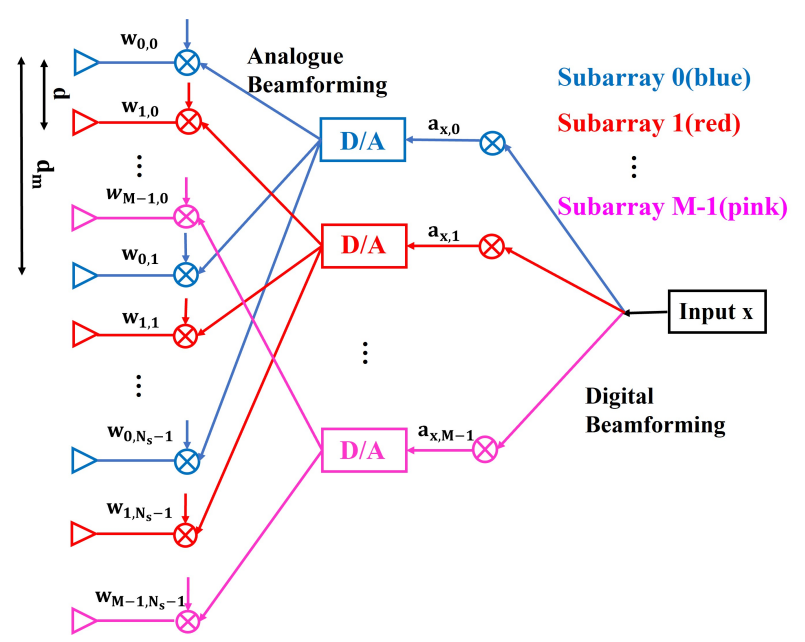

Fig. 1. A general interleaved subarray based hybrid beamforming structure.

\section{THE PROPOSED DESIGN}

Similar to the work in [23], a general digital coding technique to design $\mathrm{X}$ beams is adopted, whose coefficients for the $\mathrm{x}$-th designed beam $(\mathrm{x} \in\{0,1, \cdots, \mathrm{X}-1\})$ are given by

$$
\mathbf{w}_{\mathrm{D}, \mathrm{x}}=\left[a_{\mathrm{x}, 0}, a_{\mathrm{x}, 1}, \cdots, a_{\mathrm{x}, \mathrm{M}-1}\right],
$$

where $a_{\mathrm{x}, \mathrm{m}}(\mathrm{m} \in\{0,1, \cdots, \mathrm{M}-1\})$ are MX digital coefficients to be determined later. So the designed beam pattern for the $\mathrm{x}$-th beam in a vector form is

$$
P_{\varphi_{\mathrm{x}}}(\theta)=\sum_{\mathrm{m}=0}^{\mathrm{M}-1} a_{\mathrm{x}, \mathrm{m}}^{*} P_{\mathrm{m}}(\theta)=\sum_{\mathrm{m}=0}^{\mathrm{M}-1} a_{\mathrm{x}, \mathrm{m}}^{*} \mathbf{w}_{\mathrm{m}}^{\mathrm{H}} \mathbf{s}_{\mathrm{m}}(\theta),
$$

where $*$ denotes the conjugate operation and the sum of the sidelobe responses for the $\mathrm{X}$ beams is given by

$E_{\mathrm{s}}=\sum_{\mathrm{x}=0}^{\mathrm{X}-1} \sum_{\theta \in \Theta_{\mathrm{s}_{\mathrm{x}}}}\left|P_{\varphi_{\mathrm{x}}}(\theta)\right|^{2}=\sum_{\mathrm{x}=0}^{\mathrm{X}-1} \sum_{\theta \in \Theta_{\mathrm{s} \mathrm{x}}}\left|\sum_{\mathrm{m}=0}^{\mathrm{M}-1} a_{\mathrm{x}, \mathrm{m}}^{*} \mathbf{w}_{\mathrm{m}}^{\mathrm{H}} \mathbf{s}_{\mathrm{m}}(\theta)\right|^{2}$,

where $\Theta_{\mathrm{s}_{\mathrm{x}}}$ is the sidelobe region for the x-th beam.

Although the proposed method can be applied to arbitrary number of user beams, it is difficult to have a single general formulation to cover all the cases. Without loss of generality, we use $\mathrm{X}=\mathrm{M}=3$ as a representative example. The sum of sidelobe responses in (6) with $\mathrm{X}=3$ can be expanded as

$$
\begin{aligned}
E_{\mathrm{S}}=\sum_{\mathrm{x}=0}^{2} & \left(a_{\mathrm{x}, 0}^{*} \mathbf{w}_{0}^{\mathrm{H}} \mathbf{Q}_{\mathrm{S}_{\mathrm{x} 0}} \mathbf{w}_{0} a_{\mathrm{x}, 0}+a_{\mathrm{x}, 1}^{*} \mathbf{w}_{1}^{\mathrm{H}} \mathbf{Q}_{\mathrm{S}_{\mathrm{x} 1}} \mathbf{w}_{1} a_{\mathrm{x}, 1}\right. \\
& +a_{\mathrm{x}, 2}^{*} \mathbf{w}_{2}^{\mathrm{H}} \mathbf{Q}_{\mathrm{S}_{\mathrm{x} 2}} \mathbf{w}_{2} a_{\mathrm{x}, 2} \\
& +a_{\mathrm{x}, 0}^{*} \mathbf{w}_{0}^{\mathrm{H}} \mathbf{P}_{\mathrm{S}_{01 \mathrm{x}}} \mathbf{w}_{1} a_{\mathrm{x}, 1}+a_{\mathrm{x}, 1}^{*} \mathbf{w}_{1}^{\mathrm{H}} \mathbf{P}_{\mathrm{S}_{10 \mathrm{x}}} \mathbf{w}_{0} a_{\mathrm{x}, 0} \\
& +a_{\mathrm{x}, 0}^{*} \mathbf{w}_{0}^{\mathrm{H}} \mathbf{P}_{\mathrm{S}_{02 \mathrm{x}}} \mathbf{w}_{2} a_{\mathrm{x}, 2}+a_{\mathrm{x}, 2}^{*} \mathbf{w}_{2}^{\mathrm{H}} \mathbf{P}_{\mathrm{S}_{20 \mathrm{x}}} \mathbf{w}_{0} a_{\mathrm{x}, 0} \\
& \left.+a_{\mathrm{x}, 1}^{*} \mathbf{w}_{1}^{\mathrm{H}} \mathbf{P}_{\mathrm{S}_{12 \mathrm{x}}} \mathbf{w}_{2} a_{\mathrm{x}, 2}+a_{\mathrm{x}, 2}^{*} \mathbf{w}_{2}^{\mathrm{H}} \mathbf{P}_{\mathrm{S}_{21 \mathrm{x}}} \mathbf{w}_{1} a_{\mathrm{x}, 1}\right),
\end{aligned}
$$

with

$$
\mathbf{Q}_{\mathrm{S}_{\mathrm{xm}}}=\sum_{\theta \in \Theta_{\mathrm{s}_{\mathrm{x}}}} \mathbf{s}_{\mathrm{m}}(\theta) \mathbf{s}_{\mathrm{m}}(\theta)^{\mathrm{H}}, \mathbf{P}_{\mathrm{S}_{\mathrm{ikx}}}=\sum_{\theta \in \Theta_{\mathbf{s}_{\mathrm{x}}}} \mathbf{s}_{\mathrm{i}}(\theta) \mathbf{s}_{\mathrm{k}}(\theta)^{\mathrm{H}},
$$

\section{A. Optimisation for analogue coefficients}

With $\mathbf{w}_{\mathrm{A}}=\left[\begin{array}{lll}\mathbf{w}_{0}^{\mathrm{T}} & \mathbf{w}_{1}^{\mathrm{T}} & \mathbf{w}_{2}^{\mathrm{T}}\end{array}\right]^{\mathrm{T}}$, (7) can be rewritten as

$$
E_{\mathrm{s}}=\mathbf{w}_{\mathrm{A}}^{\mathrm{H}}\left(\mathbf{Q}_{\mathrm{S}}+\mathbf{P}_{\mathrm{S}} \tilde{\mathbf{I}}_{0}+\mathbf{R}_{\mathrm{S}} \tilde{\mathbf{I}}_{1}\right) \mathbf{w}_{\mathrm{A}},
$$

with

$$
\begin{gathered}
\mathbf{Q}_{\mathrm{S}}=\operatorname{diag}\left(\mathbf{G}_{\mathrm{S}_{0}}, \mathbf{G}_{\mathrm{S}_{1}}, \mathbf{G}_{\mathrm{S}_{2}}\right), \mathbf{P}_{\mathrm{S}}=\operatorname{diag}\left(\mathbf{H}_{\mathrm{S}_{0}}, \mathbf{H}_{\mathrm{S}_{1}}, \mathbf{H}_{\mathrm{S}_{2}}\right), \\
\mathbf{R}_{\mathrm{S}}=\operatorname{diag}\left(\mathbf{Y}_{\mathrm{S}_{0}}, \mathbf{Y}_{\mathrm{S}_{1}}, \mathbf{Y}_{\mathrm{S}_{2}}\right),
\end{gathered}
$$

$\mathbf{G}_{\mathrm{S}_{\mathrm{m}}}=\mathbf{A}_{0, \mathrm{~m}}^{\mathrm{H}} \mathbf{Q}_{\mathrm{S}_{0 \mathrm{~m}}} \mathbf{A}_{0, \mathrm{~m}}+\mathbf{A}_{1, \mathrm{~m}}^{\mathrm{H}} \mathbf{Q}_{\mathrm{S}_{1 \mathrm{~m}}} \mathbf{A}_{1, \mathrm{~m}}+\mathbf{A}_{2, \mathrm{~m}}^{\mathrm{H}} \mathbf{Q}_{\mathrm{S}_{2 \mathrm{~m}}} \mathbf{A}_{2, \mathrm{~m}}$,

$\mathbf{H}_{\mathrm{S}_{0}}=\mathbf{A}_{0,0}^{\mathrm{H}} \mathbf{P}_{\mathrm{S}_{010}} \mathbf{A}_{0,1}+\mathbf{A}_{1,0}^{\mathrm{H}} \mathbf{P}_{\mathrm{S}_{011}} \mathbf{A}_{1,1}+\mathbf{A}_{2,0}^{\mathrm{H}} \mathbf{P}_{\mathrm{S}_{012}} \mathbf{A}_{2,1}$,

$\mathbf{H}_{\mathrm{S}_{1}}=\mathbf{A}_{0,1}^{\mathrm{H}} \mathbf{P}_{\mathrm{S}_{120}} \mathbf{A}_{0,2}+\mathbf{A}_{1,1}^{\mathrm{H}} \mathbf{P}_{\mathrm{S}_{121}} \mathbf{A}_{1,2}+\mathbf{A}_{2,1}^{\mathrm{H}} \mathbf{P}_{\mathrm{S}_{122}} \mathbf{A}_{2,2}$,

$\mathbf{H}_{\mathrm{S}_{2}}=\mathbf{A}_{0,2}^{\mathrm{H}} \mathbf{P}_{\mathrm{S}_{200}} \mathbf{A}_{0,0}+\mathbf{A}_{1,2}^{\mathrm{H}} \mathbf{P}_{\mathrm{S}_{201}} \mathbf{A}_{1,0}+\mathbf{A}_{2,2}^{\mathrm{H}} \mathbf{P}_{\mathrm{S}_{202}} \mathbf{A}_{2,0}$,

$\mathbf{Y}_{\mathrm{S}_{0}}=\mathbf{A}_{0,0}^{\mathrm{H}} \mathbf{P}_{\mathrm{S}_{020}} \mathbf{A}_{0,2}+\mathbf{A}_{1,0}^{\mathrm{H}} \mathbf{P}_{\mathrm{S}_{021}} \mathbf{A}_{1,2}+\mathbf{A}_{2,0}^{\mathrm{H}} \mathbf{P}_{\mathrm{S}_{022}} \mathbf{A}_{2,2}$,

$\mathbf{Y}_{\mathrm{S}_{1}}=\mathbf{A}_{0,1}^{\mathrm{H}} \mathbf{P}_{\mathrm{S}_{100}} \mathbf{A}_{0,0}+\mathbf{A}_{1,1}^{\mathrm{H}} \mathbf{P}_{\mathrm{S}_{101}} \mathbf{A}_{1,0}+\mathbf{A}_{2,1}^{\mathrm{H}} \mathbf{P}_{\mathrm{S}_{102}} \mathbf{A}_{2,0}$,

$\mathbf{Y}_{\mathrm{S}_{2}}=\mathbf{A}_{0,2}^{\mathrm{H}} \mathbf{P}_{\mathrm{S}_{210}} \mathbf{A}_{0,1}+\mathbf{A}_{1,2}^{\mathrm{H}} \mathbf{P}_{\mathrm{S}_{211}} \mathbf{A}_{1,1}+\mathbf{A}_{2,2}^{\mathrm{H}} \mathbf{P}_{\mathrm{S}_{212}} \mathbf{A}_{2,1}$,

$$
\mathbf{A}_{\mathrm{x}, \mathrm{m}}=a_{\mathrm{x}, \mathrm{m}} \mathbf{I} \text {, }
$$

where diag [.] denotes the diagonalisation operation, $\mathbf{I}$ is a $\mathrm{N}_{\mathrm{s}} \times$ $\mathrm{N}_{\mathrm{s}}$ identity matrix, $\tilde{\mathbf{I}}_{0}$ and $\tilde{\mathbf{I}}_{1}$ are given in Section VI-A.

By guaranteeing the mainlobes of the three designed beams are in the corresponding desired directions, an intermediate formulation without any requirements for the three designed beams can be expressed as

subject to $\quad \mathbf{w}_{\mathrm{A}}^{\mathrm{H}}\left[\begin{array}{lll}\mathbf{A}_{0,0}^{\mathrm{H}} \mathbf{z}_{\mathrm{S}_{0,0}} & \mathbf{A}_{1,0}^{\mathrm{H}} \mathbf{z}_{\mathrm{S}_{1,0}} & \mathbf{A}_{2,0}^{\mathrm{H}} \mathbf{z}_{\mathrm{S}_{2,0}} \\ \mathbf{A}_{0,1}^{\mathrm{H}} \mathbf{z}_{\mathrm{S}_{0,1}} & \mathbf{A}_{1,1}^{\mathrm{H}} \mathbf{z}_{\mathrm{S}_{1,1}} & \mathbf{A}_{2,1}^{\mathrm{H}} \mathbf{z}_{\mathrm{S}_{2,1}} \\ \mathbf{A}_{0,2}^{\mathrm{H}} \mathbf{z}_{\mathrm{S}_{0,2}} & \mathbf{A}_{1,2}^{\mathrm{H}} \mathbf{z}_{\mathbf{S}_{1,2}} & \mathbf{A}_{2,2}^{\mathrm{H}} \mathbf{z}_{\mathrm{S}_{2,2}}\end{array}\right]=\left[\begin{array}{lll}1 & 1 & 1\end{array}\right]$,

with

$$
\mathbf{z}_{\mathrm{S}_{\mathrm{x}, \mathrm{m}}}=\sum_{\theta \in \Theta_{\text {main }_{\mathrm{x}}}} \mathbf{s}_{\mathrm{m}}(\theta),
$$

where $\mathbf{L}=\mathbf{V U}^{1 / 2}$, $\mathbf{U}$ denotes the diagonal matrix including all eigenvalues of $\left(\mathbf{Q}_{\mathrm{S}}+\mathbf{P}_{\mathrm{S}} \tilde{\mathbf{I}}_{0}+\mathbf{R}_{\mathrm{S}} \tilde{\mathbf{I}}_{1}\right)$ in (9), $\mathbf{V}$ the corresponding eigenvector matrix [35], [36] and $\Theta_{\text {main }_{x}}$ is the mainlobe region for the $\mathrm{x}$-th beam.

i) Equal magnitude constraint on analogue coefficients

To ensure the magnitudes of the analogue coefficients in the three subarrays to be as close as possible to each other, we consider the MinMax approach which minimises the maximum value among the $3 \mathrm{~N}_{\mathrm{s}}$ analogue coefficients for all antennas as follows

$$
\min _{\mathbf{w}_{\mathrm{A}}}\left\|\mathbf{w}_{\mathrm{A}}\right\|_{\infty},
$$

where $\|.\|_{\infty}$ is the $l_{\infty}$ norm, representing the maximum magnitude of the entries in the vector. For a given set of $a_{\mathrm{x}, \mathrm{m}}(\{\mathrm{x}, \mathrm{m}\} \in\{0,1,2\})$, using the same method as in [37], we can combine the above cost function in (15) with (13), and reach the following new formulation

$$
\begin{array}{r}
\min _{\mathbf{w}_{\mathrm{A}}} \quad J_{\mathrm{LSE}}=\frac{1-\alpha}{\mathrm{N}_{\mathrm{g}}}\left\|\mathbf{L}^{\mathrm{H}} \mathbf{w}_{\mathrm{A}}\right\|_{2}+\alpha\left\|\mathbf{w}_{\mathrm{A}}\right\|_{\infty}, \\
\text { subject to constraint in }(13),
\end{array}
$$

where $\alpha \in(0,1)$ is a trade-off factor and $\mathrm{N}_{\mathrm{g}}$ is the number of sample points in the sidelobe region for each designed beam. 
ii) Robust constraint against steering vector errors

However, the above design is based on the ideal scenario that all designed steering vectors are the same as the assumed ones. To design a beamformer robust against steering vector errors, the norm-bounded error vector $\mathbf{e}_{\mathrm{m}}$ for the $\mathrm{m}$-th subarray is introduced, and the real steering vector is indicated as $\hat{\mathbf{s}}_{\mathrm{m}}(\theta)=\mathbf{s}_{\mathrm{m}}(\theta)+\mathbf{e}_{\mathrm{m}}$, where $\mathbf{s}_{\mathrm{m}}(\theta)$ denotes the assumed steering vector of the $m$-th subarray. Similar to [38], the difference between the real and desired beam responses generated by the m-th subarray satisfies

$$
\left|\mathbf{w}_{\mathrm{m}}^{\mathrm{H}} \hat{\mathbf{s}}_{\mathrm{m}}(\theta)-\mathbf{w}_{\mathrm{m}}^{\mathrm{H}} \mathbf{s}_{\mathrm{m}}(\theta)\right|=\left|\mathbf{w}_{\mathrm{m}}^{\mathrm{H}} \mathbf{e}_{\mathrm{m}}\right| \leq \epsilon_{\mathrm{m}}\left\|\mathbf{w}_{\mathrm{m}}\right\|_{2},
$$

where $\epsilon_{\mathrm{m}}$ denotes the upper norm-bound of $\mathbf{e}_{\mathrm{m}}$ for the m-th subarray.

By combining the norm-bounded vectors $\mathbf{e}_{0}, \mathbf{e}_{1}$ and $\mathbf{e}_{2}$ into $\mathbf{e}=\left[\begin{array}{lll}\mathbf{e}_{0}^{\mathrm{T}} & \mathbf{e}_{1}^{\mathrm{T}} & \mathbf{e}_{2}^{\mathrm{T}}\end{array}\right]^{\mathrm{T}}$, the difference between the real and desired beam responses of the $\mathrm{x}$-th beam is given by

$$
\begin{aligned}
& \left|\sum_{\mathrm{m}=0}^{2} a_{\mathrm{x}, \mathrm{m}}^{*} \mathbf{w}_{\mathrm{m}}^{\mathrm{H}}\left(\hat{\mathbf{s}}_{\mathrm{m}}(\theta)-\mathbf{s}_{\mathrm{m}}(\theta)\right)\right|=\left|\sum_{\mathrm{m}=0}^{2} a_{\mathrm{x}, \mathrm{m}}^{*} \mathbf{w}_{\mathrm{m}}^{\mathrm{H}} \mathbf{e}_{\mathrm{m}}\right| \\
= & \left|\mathbf{w}_{\mathrm{A}}^{\mathrm{H}} \operatorname{diag}\left(\mathbf{A}_{\mathrm{x}, 0}^{\mathrm{H}}, \mathbf{A}_{\mathrm{x}, 1}^{\mathrm{H}}, \mathbf{A}_{\mathrm{x}, 2}^{\mathrm{H}}\right) \mathbf{e}\right| \\
\leq & \sqrt{3} \epsilon_{\mathrm{e}}\left\|\operatorname{diag}\left(\mathbf{A}_{\mathrm{x}, 0}^{\mathrm{H}}, \mathbf{A}_{\mathrm{x}, 1}^{\mathrm{H}}, \mathbf{A}_{\mathrm{x}, 2}^{\mathrm{H}}\right)\right\|_{2}\left\|\mathbf{w}_{\mathrm{A}}\right\|_{2}, \quad \forall \mathrm{x} \in\{0,1,2\},
\end{aligned}
$$

where $\epsilon_{\mathrm{e}}$ is the maximum value among $\epsilon_{0}, \epsilon_{1}$ and $\epsilon_{2}$. The following constraint can be incorporated into the design to ensure that the difference between the real and desired beam responses of the $\mathrm{x}$-th beam satisfies the following requirement

$\sqrt{3} \epsilon_{\mathrm{e}}\left\|\operatorname{diag}\left(\mathbf{A}_{\mathrm{x}, 0}^{\mathrm{H}}, \mathbf{A}_{\mathrm{x}, 1}^{\mathrm{H}}, \mathbf{A}_{\mathrm{x}, 2}^{\mathrm{H}}\right)\right\|_{2}\left\|\mathbf{w}_{\mathrm{A}}\right\|_{2} \leq \zeta, \quad \forall \mathrm{x} \in\{0,1,2\}$,

where $\zeta$ denotes the change in the magnitude of the response at the mainlobe direction given the maximum allowable steering vector error.

\section{iii) Final design}

Hence, a new optimisation problem can be formulated as

$$
\min _{\mathbf{w}_{\mathrm{A}}} J_{\mathrm{LSE}}=\frac{1-\alpha}{\mathrm{N}_{\mathrm{g}}}\left\|\mathbf{L}^{\mathrm{H}} \mathbf{w}_{\mathrm{A}}\right\|_{2}+\alpha\left\|\mathbf{w}_{\mathrm{A}}\right\|_{\infty},
$$

subject to constraint in (13),

$$
\left\|\operatorname{diag}\left(\mathbf{A}_{\mathrm{x}, 0}^{\mathrm{H}}, \mathbf{A}_{\mathrm{x}, 1}^{\mathrm{H}}, \mathbf{A}_{\mathrm{x}, 2}^{\mathrm{H}}\right)\right\|_{2}\left\|\mathbf{w}_{\mathrm{A}}\right\|_{2} \leq \frac{\sigma}{\sqrt{3}}, \quad \forall \mathrm{x} \in\{0,1,2\}
$$

where $\sigma=\zeta / \epsilon_{\mathrm{e}}$.

\section{B. Optimisation for digital coefficients}

On the other hand, if we know $\mathbf{w}_{\mathrm{A}}$, we can obtain the optimum digital coding coefficients as follows.

By combining the digital coefficients for the three beams into $\mathbf{w}_{\mathrm{D}}=\left[\mathbf{w}_{\mathrm{D}, 0}, \mathbf{w}_{\mathrm{D}, 1}, \mathbf{w}_{\mathrm{D}, 2}\right]^{\mathrm{T}}$, (7) can be written as

$$
E_{\mathrm{s}}=\mathbf{w}_{\mathrm{D}}^{\mathrm{H}} \tilde{\mathbf{M}}_{\mathrm{S}} \mathbf{w}_{\mathrm{D}} \text {, }
$$

with

$$
\begin{gathered}
\tilde{\mathbf{M}}_{\mathrm{S}}=\tilde{\mathbf{Q}}_{\mathrm{S}}+\tilde{\mathbf{P}}_{\mathrm{S}} \tilde{\mathbf{I}}_{2}+\tilde{\mathbf{R}}_{\mathrm{S}} \tilde{\mathbf{I}}_{3} \\
\tilde{\mathbf{Q}}_{\mathrm{S}}=\operatorname{diag}\left(\tilde{\mathbf{G}}_{\mathrm{S}_{00}}, \tilde{\mathbf{G}}_{\mathrm{S}_{01}}, \cdots, \tilde{\mathbf{G}}_{\mathrm{S}_{22}}\right) \\
\tilde{\mathbf{P}}_{\mathrm{S}}=\operatorname{diag}\left(\tilde{\mathbf{H}}_{\mathrm{S}_{0}}, \tilde{\mathbf{H}}_{\mathrm{S}_{1}}, \tilde{\mathbf{H}}_{\mathrm{S}_{2}}\right), \tilde{\mathbf{R}}_{\mathrm{S}}=\operatorname{diag}\left(\tilde{\mathbf{Y}}_{\mathrm{S}_{0}}, \tilde{\mathbf{Y}}_{\mathrm{S}_{1}}, \tilde{\mathbf{Y}}_{\mathrm{S}_{2}}\right),
\end{gathered}
$$

where $\tilde{\mathbf{I}}_{\mathrm{q}}(\mathrm{q} \in\{2,3,4,5\}), \tilde{\mathbf{G}}_{\mathrm{S}_{\mathrm{xm}}}, \tilde{\mathbf{H}}_{\mathrm{S}_{\mathrm{x}}}, \tilde{\mathbf{Y}}_{\mathrm{S}_{\mathrm{x}}}, \hat{\mathbf{Q}}_{\mathrm{S}_{\mathrm{xm}}}, \tilde{\mathbf{B}}_{\mathrm{S}_{\mathrm{xm}}}$, $\tilde{\mathbf{D}}_{\mathrm{S}_{\mathrm{xm}}}, \hat{\mathbf{P}}_{\mathrm{S}_{\mathrm{xm}}}$, and $\hat{\mathbf{R}}_{\mathrm{S}_{\mathrm{xm}}}$ are given in Section VI-B. Given the obtained $\mathbf{w}_{\mathrm{A}}$ in (20), the optimisation problem is given by

$$
\min _{\mathbf{w}_{\mathrm{D}}} \mathbf{w}_{\mathrm{D}}^{\mathrm{H}} \tilde{\mathbf{M}}_{\mathrm{S}} \mathbf{w}_{\mathrm{D}}, \quad \text { subject to } \mathbf{C}^{\mathrm{H}} \mathbf{w}_{\mathrm{D}}=\mathbf{f} \text {, }
$$

with

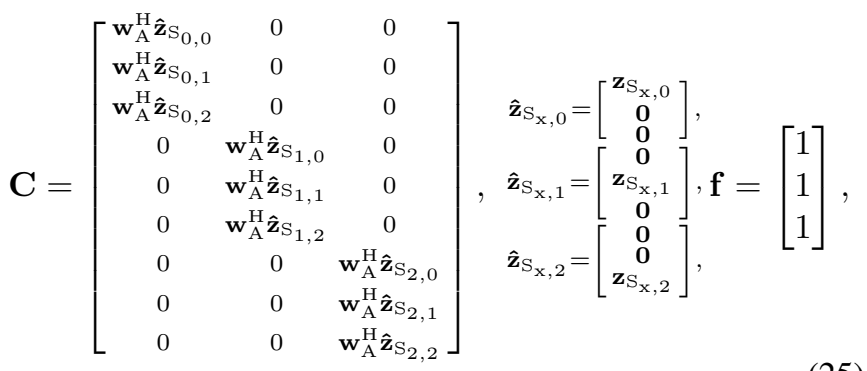

where $\mathbf{0}$ in (25) is a $\mathrm{N}_{\mathrm{s}} \times 1$ all-zero matrix.

The solution to the problem (24) is given by

$$
\mathbf{w}_{\mathrm{D}}=\tilde{\mathbf{M}}_{\mathrm{S}}^{-1} \mathbf{C}\left(\mathbf{C}^{\mathrm{H}} \tilde{\mathbf{M}}_{\mathrm{S}}^{-1} \mathbf{C}\right)^{-1} \mathbf{f} \text {. }
$$

Now alternate optimisation of $\mathbf{w}_{\mathrm{D}}$ and $\mathbf{w}_{\mathrm{A}}$ can be achieved by the following iterative process:

(1) First, to possibly increase the chance of reaching a satisfactory design result, $\mathbf{w}_{\mathrm{D}}$ is initialised with random values and $\mathrm{w}_{\mathrm{A}}$ is obtained by substituting $a_{\mathrm{x}, \mathrm{m}}(\{\mathrm{x}, \mathrm{m}\} \in$ $\{0,1,2\})$ into $(20)$.

(2) Given the obtained optimum values for $\mathbf{w}_{\mathrm{A}}$ in step (1), the closed-form solution for $\mathbf{w}_{\mathrm{D}}$ is obtained by (26).

(3) Given the obtained values of $\mathbf{w}_{D}$ in step (2), the new set of values of $\mathbf{w}_{\mathrm{A}}$ is obtained by (20) again.

(4) Repeat steps (2) and (3) until $J_{\text {LSE }}$ in (20) converges.

To see the convergence of the iterative process, we use $J_{\mathrm{LSE}}\left(\mathbf{w}_{\mathrm{A}}, \mathbf{w}_{\mathrm{D}}\right)$ to represent the cost function of this design. An important property of the cost function is that when $\mathbf{w}_{\mathrm{D}}$ is fixed, $J_{\mathrm{LSE}}\left(\mathbf{w}_{\mathrm{A}}, \mathbf{w}_{\mathrm{D}}\right)$ is a convex function with several convex constraints, while when $\mathbf{w}_{\mathrm{A}}$ is fixed, $J_{\mathrm{LSE}}\left(\mathbf{w}_{\mathrm{A}}, \mathbf{w}_{\mathrm{D}}\right)$ is a convex function with a convex constraint, as shown in (24). As a result, at each iteration, given an optimised $\mathbf{w}_{\mathrm{D}}$ in the last round, the newly optimised $\mathbf{w}_{\mathrm{A}}$ will at least not increase the value of the cost function, while given an optimised $\mathbf{w}_{\mathrm{A}}$ in the last round, the newly optimised $w_{D}$ will at least not increase the value of the cost function, i.e., the cost function will not increase during the alternate optimisation process [23].

Although the magnitudes of the coefficients in $\mathbf{w}_{\mathrm{A}}$ cannot be exactly equal after the optimisation (20), they have already been extremely close to each other and can be normalised to the same average value as follows

$$
\mathbf{w}_{\mathrm{A}}(\mathrm{n})=\frac{\mathbf{w}_{\mathrm{A}}(\mathrm{n})}{\left|\mathbf{w}_{\mathrm{A}}(\mathrm{n})\right|}\left|\overline{\mathbf{w}}_{\mathrm{A}}\right|,
$$

where $\left|\overline{\mathbf{w}}_{\mathrm{A}}\right|=\frac{1}{3 \mathrm{~N}_{\mathrm{s}}} \sum_{\mathrm{n}=0}^{3 \mathrm{~N}_{\mathrm{s}}-1}\left|\mathbf{w}_{\mathrm{A}}(\mathrm{n})\right|$ denotes the average magnitude of the analogue coefficients.

\section{DESIGN EXAMPLES}

\section{A. Parameter setting}

With $\mathrm{N}_{\mathrm{s}}=30$, design examples are provided with the interleaved and localised subarray architectures, where a fixed 
antenna spacing of $d=\frac{1}{5} \lambda$ is employed. Hence, the adjacent antenna spacing for each subarray is equal to $\frac{3 \lambda}{5}$ which is larger than $\frac{\lambda}{2}$. This also indicates the positive effect of the digital scheme, which can combine the multiple subarrays together in an effective way to suppress grating lobes.

The only difference in the design between the interleaved and localised subarray architectures is to replace the steering vector of the m-th subarray in (1) by the following:

$$
\begin{aligned}
\mathbf{s}_{\mathrm{m}}(\theta)= & {\left[e^{j 2 \pi \mathrm{mN}_{\mathrm{s}} \frac{d}{\lambda} \sin \theta}, e^{j 2 \pi\left(\mathrm{mN}_{\mathrm{s}}+1\right) \frac{d}{\lambda} \sin \theta},\right.} \\
& \left.\ldots, e^{j 2 \pi\left((\mathrm{m}+1) \mathrm{N}_{\mathrm{s}}-1\right) \frac{d}{\lambda} \sin \theta}\right]^{\mathrm{T}} .
\end{aligned}
$$

The three beam directions are $\varphi_{0}=-30^{\circ}, \varphi_{1}=0^{\circ}$ and $\varphi_{2}=35^{\circ}$ and the corresponding sidelobe regions are $\sin \Theta_{\mathrm{s}_{0}} \in[-1,-0.6] \cup[-0.4,1]\left(\sin \left(-30^{\circ}\right)=-0.5\right)$, $\sin \Theta_{\mathrm{s}_{1}} \in[-1,-0.1] \cup[0.1,1]\left(\sin \left(0^{\circ}\right)=0\right)$ and $\sin \Theta_{\mathrm{s}_{2}} \in$ $[-1,0.47] \cup[0.67,1]\left(\sin \left(35^{\circ}\right)=0.57\right)$. The number of sample points in the sidelobe and whole regions for each designed beam is $\mathrm{N}_{\mathrm{g}}=160$ and $\mathrm{N}_{\mathrm{w}}=201$, respectively.

With $\mathrm{N}_{\mathrm{s}}=30, \epsilon_{\mathrm{e}}=0.1$ is set as the upper bound on the norm of the steering vector error, which results in $\sqrt{\frac{\epsilon_{\mathrm{e}}^{2}}{\mathrm{~N}_{\mathrm{s}}}}=0.018$ of the real steering vector norm for each subarray without considering $\mathbf{w}_{\mathrm{D}}$. To give more degrees of freedom, $\zeta=0.08$ is selected to allow $8 \%$ change in the magnitude of the response and $\sigma$ is derived as $\sigma=\zeta / \epsilon_{\mathrm{e}}=0.8$.

Similar to [39], to demonstrate the robustness of this scheme, the normalised variance of beam response for the $\mathrm{x}$-th designed beam is measured as follows

$$
\operatorname{var}_{\mathrm{x}}(\theta)=\frac{1}{\mathrm{~K}} \sum_{\mathrm{k}=0}^{\mathrm{K}-1} \frac{\left|P_{\varphi_{\mathrm{x}}}^{\mathrm{k}}(\theta)-\bar{P}_{\varphi_{\mathrm{x}}}(\theta)\right|^{2}}{\left|\bar{P}_{\varphi_{\mathrm{x}}}(\theta)\right|^{2}},
$$

where $\bar{P}_{\varphi_{\mathrm{x}}}(\theta)=\frac{1}{\mathrm{~K}} \sum_{\mathrm{k}=0}^{\mathrm{K}-1} P_{\varphi_{\mathrm{x}}}^{\mathrm{k}}(\theta)$ denotes the average achieved beam response, and $P_{\varphi_{x}}^{\mathrm{k}}(\theta)$ is the beam response resultant from adding the $\mathrm{k}$-th randomly generated steering vector error satisfying the norm constraint.

\section{B. Design results}

The trade-off factor in (20) is $\alpha=0.99$. With $\mathrm{K}=2000$, the mean and instantaneous (i.e., for a particular randomly generated steering vector error) beam patterns of the three beams generated by the interleaved and localised subarray architectures are shown in Figs. 2, 3 and 4, respectively. Moreover, the change of $J_{\mathrm{LSE}}$ in (20) using the interleaved subarray architecture with respect to the iteration number and the normalised variances of beam patterns for the three designed beams are displayed in Figs. 5(a) and 5(b), respectively.

Although the variances of the zeroth beam at around 0.39, 0.5 and 1.0 are about $4.55,4.59$ and 2.22 , respectively, and the variances of the first beam at around -0.49 and -0.33 are as high as 3.06 and 2.83 , respectively, because the responses are below $-50 \mathrm{~dB}$, the variation of the beam patterns is still at an acceptable level.

The proposed design is compared to an existing design without any constraints [23], and the two other designs with two separate constraints: one with a nearly equal magnitude constraint as formulated in (16) and represented by 'Design ${ }_{0}$ '

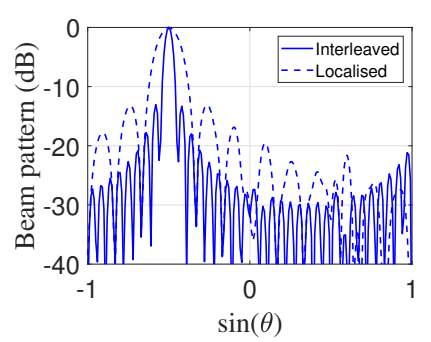

(a) Mean beam patterns.

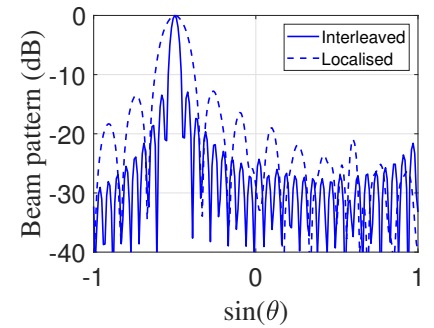

(b) Instantaneous beam patterns.
Fig. 2. The resultant patterns of the 0 th beam $\left(-30^{\circ}\right)$ for the proposed design.

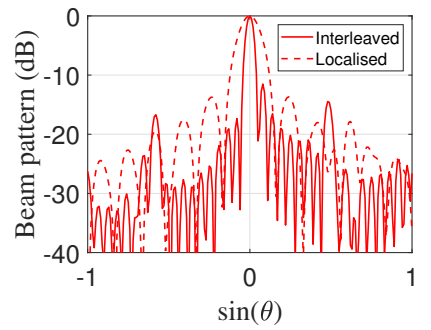

(a) Mean beam patterns.

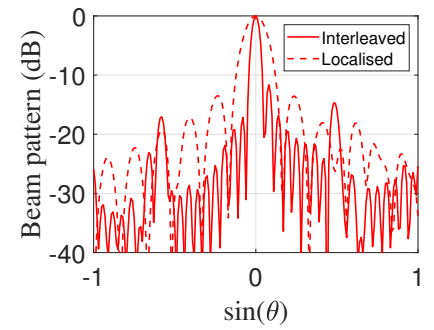

(b) Instantaneous beam patterns.
Fig. 3. The resultant patterns of the 1 st beam $\left(0^{\circ}\right)$ for the proposed design.

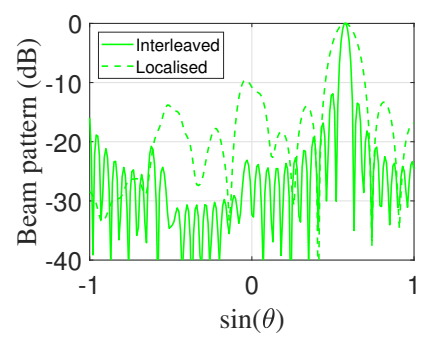

(a) Mean beam patterns.

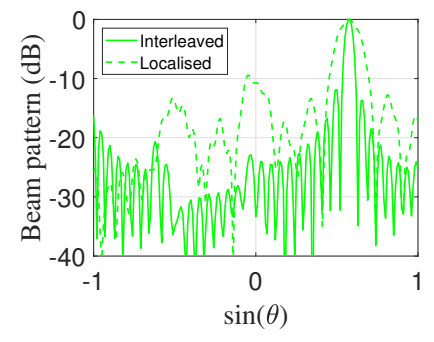

(b) Instantaneous beam patterns.
Fig. 4. The resultant patterns of the 2 nd beam $\left(35^{\circ}\right)$ for the proposed design.

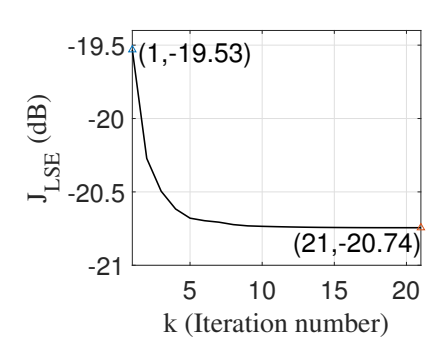

(a)

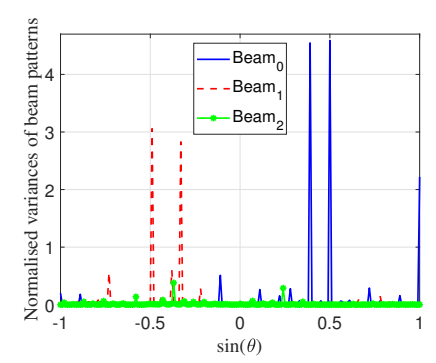

(b)
Fig. 5. (a) Cost function $J_{\mathrm{LSE}}$ in (20) with respect to the iteration number $\mathrm{k}$; (b) the normalised variances of beam patterns with $\varphi_{0}=-30^{\circ}, \varphi_{1}=0^{\circ}$ and $\varphi_{2}=35^{\circ}$ for the design with the interleaved subarray architecture.

and the other one formulated by adding the second constraint of (20) into (13), represented by 'Design ${ }_{1}$ '. 


\section{Discussions}

To compare the average sidelobe levels for all beams, the mean value for the total sidelobe responses $\bar{P}_{\mathrm{s}}$ is defined as

$$
\bar{P}_{\mathrm{s}}=\frac{1}{3 \mathrm{~N}_{\mathrm{g}}} \sum_{\mathrm{x}=0}^{2} \sum_{\mathrm{j}=0, \theta_{\mathrm{j}} \in \Theta_{\mathrm{s}_{\mathrm{x}}}}^{\mathrm{N}_{\mathrm{g}}-1}\left|P_{\varphi_{\mathrm{x}}}\left(\theta_{\mathrm{j}}\right)\right|^{2} .
$$

The overall mean variance of beam patterns is given by

$$
\bar{v}=\frac{1}{3 \mathrm{~N}_{\mathrm{w}}} \sum_{\mathrm{x}=0}^{2} \sum_{\mathrm{j}=0}^{\mathrm{N}_{\mathrm{w}}-1} \operatorname{var}_{\mathrm{x}}\left(\theta_{\mathrm{j}}\right) .
$$

To quantify the variation of the magnitudes of analogue coefficients, the normalised variance for the magnitudes of the combined analogue coefficients $\mathbf{w}_{\mathrm{A}}$ is given by

$$
\operatorname{var}_{\left|\mathbf{w}_{\mathrm{A}}\right|}=\frac{1}{3 \mathrm{~N}_{\mathrm{s}}} \sum_{\mathrm{n}=0}^{3 \mathrm{~N}_{\mathrm{s}}-1} \frac{\left\|\mathbf{w}_{\mathrm{A}}(\mathrm{n})|-| \overline{\mathbf{w}}_{\mathrm{A}}\right\|^{2}}{\left|\overline{\mathbf{w}}_{\mathrm{A}}\right|^{2}}
$$

The four parameters, including (30), (31), (32) and computation time are compared for 'Design in [23]', 'Design ${ }_{0}$ ', 'Design ${ }_{1}$ ' and the proposed design in Table I.

TABLE I

SUMMARY OF PERFORMANCES FOR DIFFERENT DESIGNS

\begin{tabular}{|c|c|c|c|c|c|}
\hline & Structure & $\bar{P}_{\mathrm{S}}(\mathrm{dB})$ & $\operatorname{var}_{\left|\mathbf{w}_{\mathrm{A}}\right|}$ & $\bar{v}$ & time $(s)$ \\
\hline \multirow{2}{*}{ Design in [23] } & Interleaved & -47.7922 & 0.8769 & 15.9600 & 26.72 \\
\cline { 2 - 6 } & Localised & -21.9728 & 0.4756 & 16.8101 & 2.75 \\
\hline \multirow{2}{*}{ Design $_{0}$} & Interleaved & -26.7840 & 0 & 0.2810 & 3.32 \\
\cline { 2 - 6 } & Localised & -18.3160 & 0 & 0.1792 & 4.27 \\
\hline \multirow{2}{*}{ Design $_{1}$} & Interleaved & -40.4851 & 0.4657 & 0.0374 & 6.07 \\
\cline { 2 - 6 } & Localised & -20.0319 & 0.2721 & 0.0104 & 3.04 \\
\hline \multirow{2}{*}{ Proposed design } & Interleaved & -25.5227 & 0 & 0.0442 & 12.57 \\
\cline { 2 - 6 } & Localised & -17.6199 & 0 & 0.0258 & 11.37 \\
\hline
\end{tabular}

Note even though the robustness is not considered in the design [23] and 'Design ${ }_{0}$ ', the maximum upper norm-bound $\epsilon_{\mathrm{e}}=0.1$ is also imposed for performance comparison.

For both the interleaved and localised architectures, the proposed design has an extra robustness property against steering vector errors with much lower mean variance of beam patterns $\bar{v}$ than 'Design ${ }_{0}$ ' at the cost of a little higher mean value for total sidelobe responses $\bar{P}_{\mathrm{s}}$. On the other hand, compared to 'Design ${ }_{1}$ ', although the mean variance of beam patterns $\bar{v}$ and the sidelobe responses $\bar{P}_{\mathrm{s}}$ of the proposed design are a little larger, the beamformer provides a uniform magnitude in its analogue coefficients due to the additional equal magnitude constraint.

As expected, $\operatorname{var}_{\left|\mathbf{w}_{\mathrm{A}}\right|}$ and $\bar{v}$ for the design in [23] are much higher than those of the other three designs; however, its $\bar{P}_{\mathrm{s}}$ is much lower because removal of the two additional constraints provides more degrees of freedom for the design. Another observation is that the interleaved architecture provides a much narrower beam than the localised one.

Finally, the effect of the trade-off factor $\alpha$ is studied briefly and the results are shown in Table II. It can be seen that with increasing values of $\alpha$, the average value of analogue magnitude $\left|\overline{\mathbf{w}}_{\mathrm{A}}\right|$ decreases at the cost of a higher value of the mean sidelobe response $\bar{P}_{\mathrm{s}}$ due to the lower demand, i.e., $(1-\alpha)$, on sidelobe suppression, but with a smaller and smaller variance value for the analogue coefficients.

TABLE II

EFFECT OF TRADE-OFF FACTOR $\alpha$ ON THE DESIGN

\begin{tabular}{|c|c|c|c|c|c|c|}
\hline$\alpha$ & Structure & 0.91 & 0.94 & 0.97 & 0.98 & 0.99 \\
\hline \multirow{2}{*}{$\bar{P}_{\mathrm{S}}(\mathrm{dB})$} & Interleaved & -26.5598 & -26.5420 & -26.5220 & -26.3243 & -25.5227 \\
\cline { 2 - 7 } & Localised & -18.9498 & -18.6679 & -18.4545 & -18.2439 & -17.6199 \\
\hline \multirow{2}{*}{$\left|\overline{\mathbf{w}}_{\mathrm{A}}\right|$} & Interleaved & 0.2264 & 0.2260 & 0.2257 & 0.2215 & 0.0084 \\
\cline { 2 - 7 } & Localised & 0.0063 & 0.0053 & 0.0051 & 0.0050 & 0.0049 \\
\hline \multirow{2}{*}{$\operatorname{var}\left|\mathbf{w}_{\mathrm{A}}\right|$} & Interleaved & $7.1 \times 10^{-15}$ & $1.0 \times 10^{-15}$ & $1.8 \times 10^{-16}$ & $1.2 \times 10^{-16}$ & $6.5 \times 10^{-17}$ \\
\cline { 2 - 7 } & Localised & 0.0237 & 0.0169 & 0.0107 & $8.2 \times 10^{-12}$ & $1.5 \times 10^{-13}$ \\
\hline
\end{tabular}

\section{CONClusion}

A multi-beam multiplexing design method considering two practical application constraints has been proposed based on the sub-aperture subarray architectures. First, all the analogue antenna coefficients share the same magnitude, so that only phase changes are needed in its implementation; then, robustness of the system against various steering vector errors was considered by introducing a norm-bounded error. As demonstrated by design examples, a successful design has been achieved by the proposed solution.

\section{APPENDIX}

\section{A. Appendix for Section III-A}

To transform (7) into a quadratic form in (9) for convex optimisation of $\mathbf{w}_{\mathrm{A}}$ straightforwardly, $\tilde{\mathbf{I}}_{0}$ and $\tilde{\mathbf{I}}_{1}$ are given by

$$
\tilde{\mathbf{I}}_{0}=\tilde{\mathbf{I}}_{1}^{\mathrm{T}}=\left[\begin{array}{lll}
\mathbf{0} & \mathbf{I} & \mathbf{0} \\
\mathbf{0} & \mathbf{0} & \mathbf{I} \\
\mathbf{I} & \mathbf{0} & \mathbf{0}
\end{array}\right],
$$

where $\mathbf{0}$ and $\mathbf{I}$ are $\mathrm{N}_{\mathrm{s}} \times \mathrm{N}_{\mathrm{s}}$ all-zero and identity matrices, respectively.

\section{B. Appendix for Section III-B}

Similar to $\tilde{\mathbf{I}}_{0}$ and $\tilde{\mathbf{I}}_{1}, \tilde{\mathbf{I}}_{2}$ and $\tilde{\mathbf{I}}_{3}$ are given by

$$
\tilde{\mathbf{I}}_{2}=\tilde{\mathbf{I}}_{3}^{\mathrm{T}}=\left[\begin{array}{lllllllll}
0 & 1 & 0 & 0 & 0 & 0 & 0 & 0 & 0 \\
0 & 0 & 1 & 0 & 0 & 0 & 0 & 0 & 0 \\
1 & 0 & 0 & 0 & 0 & 0 & 0 & 0 & 0 \\
0 & 0 & 0 & 0 & 1 & 0 & 0 & 0 & 0 \\
0 & 0 & 0 & 0 & 0 & 1 & 0 & 0 & 0 \\
0 & 0 & 0 & 1 & 0 & 0 & 0 & 0 & 0 \\
0 & 0 & 0 & 0 & 0 & 0 & 0 & 1 & 0 \\
0 & 0 & 0 & 0 & 0 & 0 & 0 & 0 & 1 \\
0 & 0 & 0 & 0 & 0 & 0 & 1 & 0 & 0
\end{array}\right],
$$

for the optimisation of $\mathbf{w}_{\mathrm{D}}$. Moreover, the symbols which constitute $\tilde{\mathbf{Q}}_{\mathrm{S}}, \tilde{\mathbf{P}}_{\mathrm{S}}$ and $\tilde{\mathbf{R}}_{\mathrm{S}}$ in (22) are given as follows

$$
\begin{aligned}
& \tilde{\mathbf{G}}_{\mathrm{S}_{\mathrm{xm}}}=\mathbf{w}_{\mathrm{A}}^{\mathrm{H}} \hat{\mathbf{Q}}_{\mathrm{S}_{\mathrm{xm}}} \mathbf{w}_{\mathrm{A}} \text {, } \\
& \tilde{\mathbf{H}}_{\mathrm{S}_{\mathrm{x}}}=\operatorname{diag}\left(\tilde{\mathbf{B}}_{\mathrm{S}_{\mathrm{x} 0}}, \tilde{\mathbf{B}}_{\mathrm{S}_{\mathrm{x} 1}}, \tilde{\mathbf{B}}_{\mathrm{S}_{\mathrm{x} 2}}\right), \tilde{\mathbf{Y}}_{\mathrm{S}_{\mathrm{x}}}=\operatorname{diag}\left(\tilde{\mathbf{D}}_{\mathrm{S}_{\mathrm{x} 0}}, \tilde{\mathbf{D}}_{\mathrm{S}_{\mathrm{x} 1}}, \tilde{\mathbf{D}}_{\mathrm{S}_{\mathrm{x} 2}}\right) \text {, } \\
& \hat{\mathbf{Q}}_{\mathrm{S}_{\mathrm{x} 0}}=\left[\begin{array}{ccc}
\mathbf{Q}_{\mathrm{S}_{\mathrm{x} 0}} & \mathbf{0} & \mathbf{0} \\
\mathbf{0} & \mathbf{0} & \mathbf{0} \\
\mathbf{0} & \mathbf{0} & \mathbf{0}
\end{array}\right], \hat{\mathbf{Q}}_{\mathrm{S}_{\mathrm{x} 1}}=\left[\begin{array}{ccc}
\mathbf{0} & \mathbf{0} & \mathbf{0} \\
\mathbf{0} & \mathbf{Q}_{\mathrm{S}_{\mathrm{x} 1}} & \mathbf{0} \\
\mathbf{0} & \mathbf{0} & \mathbf{0}
\end{array}\right], \hat{\mathbf{Q}}_{\mathrm{S}_{\mathrm{x} 2}}=\left[\begin{array}{lll}
\mathbf{0} & \mathbf{0} & \mathbf{0} \\
\mathbf{0} & \mathbf{0} & \mathbf{0} \\
\mathbf{0} & \mathbf{0} & \mathbf{Q}_{\mathrm{S}_{\mathrm{x} 2}}
\end{array}\right], \\
& \tilde{\mathbf{B}}_{\mathrm{S}_{\mathrm{x} 0}}=\mathbf{w}_{\mathrm{A}}^{\mathrm{H}} \hat{\mathbf{P}}_{\mathrm{S}_{\mathrm{x} 0}} \tilde{\mathbf{I}}_{4} \mathbf{w}_{\mathrm{A}}, \quad \tilde{\mathbf{D}}_{\mathrm{S}_{\mathrm{x} 0}}=\mathbf{w}_{\mathrm{A}}^{\mathrm{H}} \hat{\mathbf{R}}_{\mathrm{S}_{\mathrm{x} 0}} \tilde{\mathbf{I}}_{1} \mathbf{w}_{\mathrm{A}}, \\
& \tilde{\mathbf{B}}_{\mathrm{S}_{\mathrm{x} 1}}=\mathbf{w}_{\mathrm{A}}^{\mathrm{H}} \hat{\mathbf{P}}_{\mathrm{S}_{\mathrm{x} 1}} \tilde{\mathbf{I}}_{5} \mathbf{w}_{\mathrm{A}}, \quad \tilde{\mathbf{D}}_{\mathrm{S}_{\mathrm{x} 1}}=\mathbf{w}_{\mathrm{A}}^{\mathrm{H}} \hat{\mathbf{R}}_{\mathrm{S}_{\mathrm{x} 1}} \tilde{\mathbf{I}}_{4} \mathbf{w}_{\mathrm{A}} \text {, } \\
& \tilde{\mathbf{B}}_{\mathrm{S}_{\mathrm{x} 2}}=\mathbf{w}_{\mathrm{A}}^{\mathrm{H}} \hat{\mathbf{P}}_{\mathrm{S}_{\mathrm{x} 2}} \tilde{\mathbf{I}}_{0} \mathbf{w}_{\mathrm{A}}, \quad \tilde{\mathbf{D}}_{\mathrm{S}_{\mathrm{x} 2}}=\mathbf{w}_{\mathrm{A}}^{\mathrm{H}} \hat{\mathbf{R}}_{\mathrm{S}_{\mathrm{x} 2}} \tilde{\mathbf{I}}_{5} \mathbf{w}_{\mathrm{A}} \text {, }
\end{aligned}
$$




$$
\begin{aligned}
& \hat{\mathbf{P}}_{\mathrm{S}_{\mathrm{x} 0}}=\left[\begin{array}{ccc}
\mathbf{P}_{\mathrm{S}_{01 \times}} & \mathbf{0} & \mathbf{0} \\
\mathbf{0} & \mathbf{0} & \mathbf{0} \\
\mathbf{0} & \mathbf{0} & \mathbf{0}
\end{array}\right], \hat{\mathbf{P}}_{\mathrm{S}_{\mathrm{x} 1}}=\left[\begin{array}{ccc}
\mathbf{0} & \mathbf{0} & \mathbf{0} \\
\mathbf{0} & \mathbf{P}_{\mathrm{S}_{12 \mathrm{x}}} & \mathbf{0} \\
\mathbf{0} & \mathbf{0} & \mathbf{0}
\end{array}\right], \hat{\mathbf{P}}_{\mathrm{S}_{\mathrm{x} 2}}=\left[\begin{array}{ccc}
\mathbf{0} & \mathbf{0} & \mathbf{0} \\
\mathbf{0} & 0 & \mathbf{0} \\
\mathbf{0} & \mathbf{0} & \mathbf{P}_{\mathrm{S}_{20 \times}}
\end{array}\right], \\
& \hat{\mathbf{R}}_{\mathrm{S}_{\mathrm{x} 0}}=\left[\begin{array}{ccc}
\mathbf{P}_{\mathrm{S}_{02 \times}} & 0 & 0 \\
0 & 0 & 0 \\
0 & 0 & 0
\end{array}\right], \hat{\mathbf{R}}_{\mathrm{S}_{\mathrm{x} 1}}=\left[\begin{array}{ccc}
0 & 0 & 0 \\
0 & \mathbf{P}_{\mathrm{S}_{10 \times}} & 0 \\
0 & \mathbf{0} & \mathbf{0}
\end{array}\right], \hat{\mathbf{R}}_{\mathrm{S}_{\mathrm{x} 2}}=\left[\begin{array}{ccc}
0 & 0 & 0 \\
0 & 0 & 0 \\
0 & 0 & \mathbf{P}_{\mathrm{S}_{21}}
\end{array}\right], \\
& \tilde{\mathbf{I}}_{4}=\left[\begin{array}{lll}
0 & \mathbf{I} & 0 \\
\mathbf{I} & 0 & 0 \\
0 & 0 & \mathrm{I}
\end{array}\right], \quad \tilde{\mathbf{I}}_{5}=\left[\begin{array}{lll}
\mathbf{l} & 0 & 0 \\
0 & 0 & \mathrm{I} \\
0 & \mathrm{I} & 0
\end{array}\right],
\end{aligned}
$$

where $\tilde{\mathbf{I}}_{4}$ and $\tilde{\mathbf{I}}_{5}$ are similar to $\tilde{\mathbf{I}}_{0}$ and $\tilde{\mathbf{I}}_{1}$ in (33).

\section{REFERENCES}

[1] F. Boccardi, R. W. Heath, A. Lozano, T. L. Marzetta, and P. Popovski, "Five disruptive technology directions for 5G," IEEE Commun. Mag., vol. 52, no. 2, pp. 74-80, Feb. 2014.

[2] W. Roh, J. Seol, J. Park, et al., "Millimeter-wave beamforming as an enabling technology for $5 \mathrm{G}$ cellular communications: Theoretical feasibility and prototype results," IEEE Commun. Mag., vol. 52, no. 2, pp. 106-113, Feb. 2014.

[3] S. Han, C. I. I, Z. Xu, and C. Rowell, "Large-scale antenna systems with hybrid analog and digital beamforming for millimeter wave 5G," IEEE Commun. Mag., vol. 53, no. 1, pp. 186-194, Jan. 2015.

[4] A. F. Molisch, V. V. Ratnam, S. Han, et al., "Hybrid beamforming for massive MIMO: A survey," IEEE Commun. Mag., vol. 55, no. 9, pp. 134-141, Sep. 2017.

[5] F. Sohrabi and W. Yu, "Hybrid analog and digital beamforming for mmWave OFDM large-scale antenna arrays," IEEE J. Sel. Areas Commun., vol. 35, no. 7, pp. 1432-1443, Jul. 2017.

[6] S. A. Busari, K. M. S. Huq, S. Mumtaz, et al., "Generalized hybrid beamforming for vehicular connectivity using THz massive MIMO," IEEE Trans. Veh. Technol., vol. 68, no. 9, pp. 8372-8383, Sep. 2019.

[7] K. Satyanarayana, M. El-Hajjar, P. Kuo, et al., "Hybrid beamforming design for full-duplex millimeter wave communication," IEEE Trans. Veh. Technol., vol. 68, no. 2, pp. 1394-1404, Feb. 2019.

[8] S. Payami, M. Ghoraishi, M. Dianati, et al., "Hybrid beamforming with a reduced number of phase shifters for massive MIMO systems," IEEE Trans. Veh. Technol., vol. 67, no. 6, pp. 4843-4851, Jun. 2018.

[9] Z. Zheng and H. Gharavi, "Spectral and energy efficiencies of millimeter wave MIMO with configurable hybrid precoding," IEEE Trans. Veh. Technol., vol. 68, no. 6, pp. 5732-5746, Jun. 2019.

[10] M. M. Molu, P. Xiao, M. Khalily, et al., "Low-complexity and robust hybrid beamforming design for multi-antenna communication systems," IEEE Trans. Wireless Commun., vol. 17, no. 3, pp. 1445-1459, Mar. 2018.

[11] A. Li and C. Masouros, "Energy-efficient SWIPT: From fully digital to hybrid analog-digital beamforming," IEEE Trans. Veh. Technol., vol. 67, no. 4, pp. 3390-3405, Apr. 2018.

[12] V. Venkateswaran and A. van der Veen, "Analog beamforming in MIMO communications with phase shift networks and online channel estimation,” IEEE Trans. Signal Process., vol. 58, no. 8, pp. 4131-4143, Aug. 2010.

[13] X. Huang and Y. Guo, "Frequency-domain AoA estimation and beamforming with wideband hybrid arrays," IEEE Trans. Wireless Commun., vol. 10, no. 8, pp. 2543-2553, Aug. 2011.

[14] T. S. Rappaport, S. Sun, R. Mayzus, et al., "Millimeter wave mobile communications for 5G cellular: It will work!," IEEE Access, vol. 1, pp. 335-349, May 2013.

[15] J. A. Zhang, X. Huang, V. Dyadyuk, and Y. J. Guo, "Massive hybrid antenna array for millimeter-wave cellular communications," IEEE Wirel. Commun., vol. 22, no. 1, pp. 79-87, Feb. 2015.

[16] Y. Guo, X. Huang, and V. Dyadyuk, "A hybrid adaptive antenna array for long-range mm-Wave communications," IEEE Antennas Propag. Mag., vol. 54, no. 2, pp. 271-282, Apr. 2012.

[17] S. Fujio, C. Kojima, T. Shimura, et al., "Robust beamforming method for SDMA with interleaved subarray hybrid beamforming," in Proc. IEEE Annual International Symposium on Personal, Indoor, and Mobile Radio Communications (PIMRC), Valencia, Spain, Sep. 2016, pp. 1-5.

[18] T. Shimura, T. Ohshima, H. Ashida, et al., "Millimeter-wave TX phased array with phase adjusting function between transmitters for hybrid beamforming with interleaved subarrays," in Proc. European Microwave Conference (EuMC), London, UK, Oct. 2016, pp. 1572-1575.

[19] P. Rocca, R. Haupt, and A. Massa, "Sidelobe reduction through element phase control in uniform subarrayed array antennas," IEEE Antennas Wireless Propag. Lett., vol. 8, pp. 437-440, Feb. 2009.
[20] Z. Li, A. Honda, T. Shimura, et al., "Multi-user mmWave communication by interleaved beamforming with inter-subarray coding," in Proc. IEEE Annual International Symposium on Personal, Indoor, and Mobile Radio Communications (PIMRC), Montreal, QC, Canada, Oct. 2017, pp. $1-6$.

[21] M. Shimizu, A. Honda, S. Ishikawa, et al., "Millimeter-wave beam multiplexing method using hybrid beamforming," in Proc. IEEE Annual International Symposium on Personal, Indoor, and Mobile Radio Communications (PIMRC), Valencia, Spain, Sep. 2016, pp. 1-6.

[22] M. Shimizu, "Millimeter-wave beam multiplexing method using subarray type hybrid beamforming of interleaved configuration with intersubarray coding," Int. J. Wirel. Inf. Netw., vol. 24, no. 3, pp. 217-224, Sep. 2017.

[23] J. Zhang, W. Liu, C. Gu, S. S. Gao, and Q. Luo, "Multi-beam multiplexing design for arbitrary directions based on the interleaved subarray architecture," IEEE Trans. Veh. Technol., vol. 69, no. 10, pp. 11220-11232, Oct. 2020.

[24] B. Zhang, W. Liu, Y. Li, X. Zhao, and C. Wang, "Directional modulation design under a constant magnitude constraint for weight coefficients," IEEE Access, vol. 7, pp. 154711-154718, Oct. 2019.

[25] C. Lu, W. Sheng, Y. Han, and X. Ma, "A novel adaptive phase-only beamforming algorithm based on semidefinite relaxation," in Proc. IEEE International Symposium on Phased Array Systems and Technology, Waltham, MA, USA, Oct. 2013, pp. 617-621.

[26] P. J. Kajenski, "Phase only antenna pattern notching via a semidefinite programming relaxation," IEEE Trans. Antennas Propag., vol. 60, no. 5, pp. 2562-2565, May 2012.

[27] Ö. T. Demir and T. E. Tuncer, "Optimum discrete phase-only transmit beamforming with antenna selection," in Proc. European Signal Processing Conference (EUSIPCO), Lisbon, Portugal, Sep. 2014, pp. $1282-1286$

[28] R. V. Gatti, L. Marcaccioli, and R. Sorrentino, "A novel phase-only method for shaped beam synthesis and adaptive nulling," in Proc. IEEE European Microwave Conference, Munich, Germany, 2003, pp. 739742.

[29] I. D. Olin, "Flat-top sector beams using only array element phase weighting: A metaheuristic optimization approach," Tech. rep., Naval Res. Lab., Washington, DC, USA, Oct. 2012.

[30] J. Song, J. Choi, and D. J. Love, "Codebook design for hybrid beamforming in millimeter wave systems," in Proc. IEEE International Conference on Communications, London, UK, 2015, pp. 1298-1303.

[31] S. A. Vorobyov, A. B. Gershman, Z. Luo, and N. Ma, "Adaptive beamforming with joint robustness against mismatched signal steering vector and interference nonstationarity," IEEE Signal Process. Lett., vol. 11, no. 2, pp. 108-111, Feb. 2004.

[32] S. Kim, A. Magnani, A. Mutapcic, S. P. Boyd, and Z. Luo, "Robust beamforming via worst-case SINR maximization," IEEE Trans. Signal Process., vol. 56, no. 4, pp. 1539-1547, Apr. 2008.

[33] S. A. Vorobyov, A. B. Gershman, and Z. Luo, "Robust adaptive beamforming using worst-case performance optimization: a solution to the signal mismatch problem," IEEE Trans. Signal Process., vol. 51, no. 2, pp. 313-324, Feb. 2003.

[34] S. Shahbazpanahi, A. B. Gershman, Z. Luo, and K. M. Wong, "Robust adaptive beamforming for general-rank signal models," IEEE Trans. Signal Process., vol. 51, no. 9, pp. 2257-2269, Sep. 2003.

[35] Y. Zhao, W. Liu, and R. J. Langley, "Adaptive wideband beamforming with frequency invariance constraints," IEEE Trans. Antennas Propag., vol. 59, no. 4, pp. 1175-1184, Apr. 2011.

[36] M. Grant and S. Boyd, "CVX: Matlab software for disciplined convex programming, version 2.1," http://cvxr.com/cvx, Mar. 2014.

[37] J. Zhang, W. Liu, C. Gu, S. S. Gao, and Q. Luo, "Dual-beam multiplexing under an equal magnitude constraint based on a hybrid beamforming structure," in Proc. IEEE Annual International Symposium on Personal, Indoor and Mobile Radio Communications (PIMRC), London, UK, Aug. 2020, pp. 1-5.

[38] M. Rubsamen and M. Pesavento, "Maximally robust capon beamformer," IEEE Trans. Signal Process., vol. 61, no. 8, pp. 2030-2041, Apr. 2013.

[39] M. B. Hawes and W. Liu, "Sparse array design for wideband beamforming with reduced complexity in tapped delay-lines," IEEE/ACM Trans. Audio, Speech, Language Process., vol. 22, no. 8, pp. 1236-1247, Aug. 2014. 